J. Lake Sci. (湖泊科学), $2006, \mathbf{1 8}(4): 419-424$

http:// www. jlakes. org. E-mail: jlakes@ niglas. ac.cn

(c) 2006 by Journal of Lake Sciences

\title{
利用流式细胞仪研究温度对两种藻竞争的影响
}

\author{
谭 啸 ${ }^{1,2}$, 孔繁翔 ${ }^{1 * *}$, 曹焕生 ${ }^{1,3}$, 史小丽 ${ }^{1}$, 于 洋 ${ }^{1}$, 张 民 民 $^{1,3}$ \\ (1. 中国科学院南京地理与湖泊研究所, 南京 210008) \\ (2. 南京师范大学生命科学学院, 南京 210097) \\ (3. 中国科学院研究生院, 北京 100039)
}

摘 要: 为研究温度对微囊藻优势形成的影响, 本试验利用流式细胞仪研究了不同温度时铜绿微囊藻 (Microcystis aerugi$n o s a$ ) 与蛋白核小球藻 (Chlorella pyrenoidosa) 间的竞争关系, 在单独培养和混合培养条件下分别对两种藻细胞密度比值和 细胞内叶绿素 $\mathrm{a}(\mathrm{Chla})$ 苂光强度进行了测定. 结果表明在本试验条件下, 随着温度的升高 $\left(18^{\circ} \mathrm{C}, 25^{\circ} \mathrm{C}\right.$ 和 $\left.32^{\circ} \mathrm{C}\right)$, 混合培养 组中微囊藻优势逐渐明显, 小球藻 Chla 苂光强度随着温度的升高而升高, 微囊藻 Chla 苂光强度随着温度的升高却有所降 低. 说明在光照适宜、高营养盐的水体中, 较高的温度对铜绿微囊藻成为优势种具有明显的促进作用, 进而影响着微囊藻 水华的形成.

关键词: 流式细胞仪;温度; 铜绿微囊藻;蛋白核小球藻; 竞争

\section{Influences of temperatures on the competition between two species of algae assayed by flow cytometry}

TAN Xiao ${ }^{1,2}$, KONG Fanxiang ${ }^{1{ }^{* *}}$, CAO Huansheng ${ }^{1,3}$, SHI Xiaoli ${ }^{1}$, YU Yang ${ }^{1} \&$ ZHANG Min ${ }^{1,3}$

(1:Nanjing Institute of Geography and Limnology, Chinese Academy of Sciences, Nanjing 210008, P. R. China)

(2: College of Life Science, Nanjing Normal University, Nanjing 210097, P. R. China)

(3: Graduate School, Chinese Academy of Sciences, Beijing 100039, P. R. China)

Abstract:To detect the influences of different temperatures on Microcystis aeruginosa dominance, this experiment researched competition between Microcystis aeruginosa and Chlorella pyrenoidosa under different temperatures by flow cytometry, cell density ratio and chlorophyll-a fluorescence intensity in two species of algae cell were measured. Results displayed under this experimental condition Microcystis aeruginosa dominance became more evident together with temperature rised $\left(18^{\circ} \mathrm{C}, 25^{\circ} \mathrm{C}\right.$ and $\left.32^{\circ} \mathrm{C}\right)$. Higher temperature enhanced chlorophyll-a fluorescence intensity in Chlorella pyrenoidosa cell but reduced chlorophyll-a fluorescence intensity in Microcystis aeruginosa cell. This experiment indicated under suitable light and high nutrition salt condition, higher temperature promoted Microcystis aeruginosa dominance obviously and influenced water bloom forming.

Keywords: Flow cytometry; temperature; Microcystis aeruginosa; Chlorella pyrenoidosa; competition

太湖等富营养化湖泊中铜绿微囊藻成为优势种群并形成水华,对湖泊环境、水产养殖以及饮用水安全 造成了巨大危害 ${ }^{[1,2]}$. 为探明微囊藻水华的形成机理, 人们对太湖水体进行了长期的野外跟踪观测和室内 模拟研究. 孔繁翔等人总结出微囊藻水华形成大致分为冬眠、复苏、生物量增加 (生长)、上浮及聚集四个阶 段, 并认为每个阶段蓝藻的生理特性和环境主导因子有所不同 ${ }^{[3]}$. 多年的观测资料也显示太湖中微囊藻并 非长年都占优势, 而仅仅在 3、4 月份以后随着水温的升高, 原本在低温时占优势的硅藻和绿藻逐渐减少, 微

* 国家重点基础研究发展计划(2002CB412305)、国家自然科学基金(40471045)、中国科学院百人计划和南京地理与 湖泊研究所所长基金联合资助. 2005-09-23 收稿;2005-12-22 收修改稿. 谭啸,男,1981 年生,硕士研究生;Email:biotan@163.com.

** 通讯联系人;E-mail: fxkong@ niglas. ac.cn. 
囊藻逐渐成为优势种 ${ }^{[1]}$. 基于这一现象, 本试验研究不同温度时蓝藻水华常见种——铜绿微囊藻和湖泊中 常见的绿藻——蛋白核小球藻间的竞争关系, 探讨微囊藻在与其他藻类竞争中逐渐形成优势的机制.

有关藻类竞争的试验已有一些报道. Holm \& Armstrong 研究了硅藻和微囊藻间的相互作用 ${ }^{[4]}$; Akira \& Kuwata 研究了氨盐供应速率对蓝藻和绿藻竞争的影响 ${ }^{[5]}$; Kaoru Takeya 等研究了恒化培养条件下微囊藻与 栅藻间的竞争关系 ${ }^{[6]}$; 陈德辉等研究了铜绿微囊藻与栅藻间的相互作用并计算了竞争参数 ${ }^{[7]}$. 本试验选用 的流式细胞仪 (Flow Cytometry, FCM) 在以前的淡水藻类竞争研究中使用较少 ${ }^{[8]}$, 较之传统的显微计数方法 极大地减小了实验误差并获取了以前难以获得的数据. Huisman 等曾利用 FCM 研究了富营养化水体中淡 水浮游生物的竞争试验, 建立了有效的光竞争模型 ${ }^{[9]}$. 本试验利用 FCM 技术, 混合培养不同藻类, 研究不同 温度时两种藻在共存条件下的竞争过程,这在国内外尚未见报道.

\section{1 材料与方法}

\section{1 试验材料}

铜绿微囊藻 (Microcystis aeruginosa) 和蛋白核小球藻(Chlorella pyrenoidosa) 藻种购自中科院水生所淡水 藻种库. 培养基耖选的预试验显示在 $\mathrm{BG}-11$ 培养基中纯培养的两种藻生长状况最好, 所以竞争试验选用 BG - 11 培养基,在接种前纯藻种均经新鲜的 BG - 11 培养基活化一周.

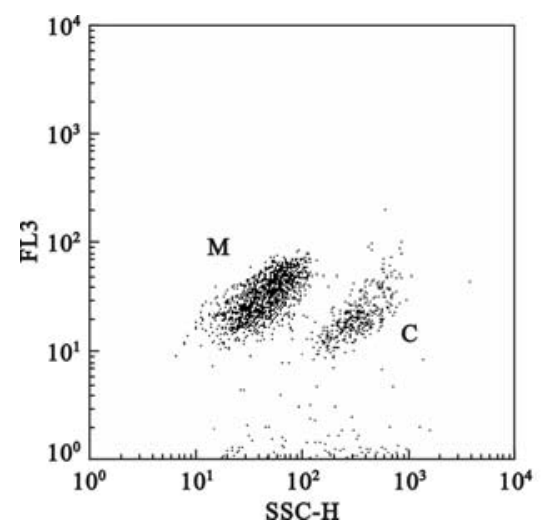

图 1 微囊藻与小球藻的二维分布图 ( $\mathrm{M}$ :微囊藻; $\mathrm{C}$ : 小球藻)

Fig. $12-\mathrm{D}$ dotplot of Microcystis aeruginosa and Chlorelia pyrenoidosa

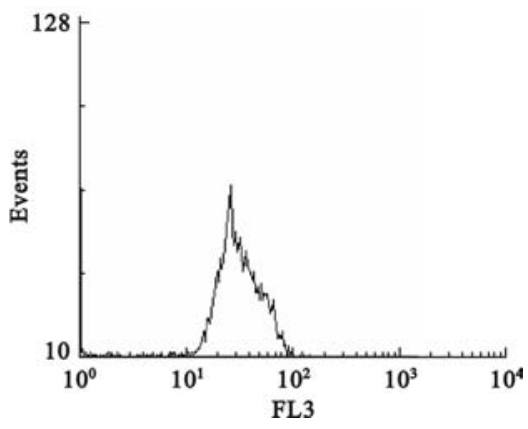

图 2 藻细胞内 Chla 的苂光强度

Fig. 2 Chla intensity in algae cell

\section{( M: Microcystis aeruginosa; $\mathrm{C}:$ Chlorella pyrenoidosa)}

\section{2 试验方法}

试验中设置铜绿微囊藻、蛋白核小球藻的纯培养对照以及两种藻 1: 1 混合培养组三个处理, 各个处理 设置三个平行, 试验分别在 $18^{\circ} \mathrm{C} 、 25^{\circ} \mathrm{C} 、 32^{\circ} \mathrm{C}$ 三个温度进行, 共 27 个样本. 选用 $500 \mathrm{ml}$ 三角瓶装人 $150 \mathrm{ml}$ 培 养基, 接种后起始藻密度均为 $2 \times 10^{5} \mathrm{cells} / \mathrm{ml}$ (混合培养的两种藻密度各为 $2 \times 10^{5} \mathrm{cells} / \mathrm{ml}$ ). 培养时光照强 度均为 $2000 \mathrm{~lx}$, 光周期为 $12 \mathrm{~h}: 12 \mathrm{~h}$.

从接种之日起每隔一天从各个三角瓶取藻液, 以测定在纯培养和混合培养条件下微囊藻和小球藻的藻 细胞密度比值 (藻细胞密度比值指每 $\mathrm{ml}$ 培养液中两种藻各自细胞数的比值) 和各自细胞内的 Chla 菼光 强度.

1.2.1 藻密度比值的测定 取纯培养对照组和混合培养组藻液各 $1 \mathrm{ml}$, 通过 FCM (FACSVantageSE BectonDickinson, 美国) 在 $488 \mathrm{~nm}, 200 \mathrm{~mW}$ 功率激光 (Coherent Innova, 美国) 激发下的 SSC-H ( Side-angle scatter height, 侧向散射角大小反映细胞对激光束的侧向散射特性) 和 FL3 (Fluorescence channel 3, Chla 菼光强 度) 构建二维坐标图定量检测混合培养组中微囊藻和小球藻各自的细胞密度 (图 1). 用 Cellquest 7.5.3 软 件对藻细胞密度比值进行统计分析并用 WinMDI 2.9 软件作图. FCM 检测的 FL3 以及 SSC 大小均为一相对 值, 坐标刻度以指数排列, 其绝对值可以结合内参计算获得. 
1.2.2 Chla 荧光强度的测定 在每次测量前用 Nile 标准小球(Caltag, 美国) 把 $200 \mathrm{~mW}, 488 \mathrm{~nm}$ 激光激发后 FL3 通道 $(650 \mathrm{~nm}-670 \mathrm{~nm})$ 的信号强度校正为 456 , 使每次检测的苂光强度具有可比性. 取 $1 \mathrm{ml}$ 纯培养和混 合培养的藻液, 在 FCM 上分别检测经 $488 \mathrm{~nm}$ 激光激发后微囊藻和小球藻在 FL3 通道各自细胞内 Chla 的菼 光强度 (图 2), 图中横坐标 FL3 表示 Chla 苂光强度, 纵坐标表示某一苂光强度的出现频率. 对检测结果运 用 SPSS 12.0 软件进行统计分析.

\section{2 结果与讨论}

\section{1 不同温度时的藻密度比值}

温度对于藻类竞争有显著的影响, 在较低温度 $\left(18^{\circ} \mathrm{C}\right)$ 微囊藻与小球藻的密度比值先下降后上升, 在较 高温度 $\left(25^{\circ} \mathrm{C}\right.$ 和 $32^{\circ} \mathrm{C}$ ) 两者密度比值是一直上升的趋势 (图 3), 并且在 $32^{\circ} \mathrm{C}$ 微囊藻优势更加明显. 原因主要 在于微囊藻是喜温藻类, 其最适生长温度为 $20-35^{\circ} \mathrm{C}$, 而小球藻的最适生长温度在 $24-28^{\circ} \mathrm{C}^{[10]}$. 微囊藻含 有 Chla 和藻蓝素两种色素, 可利用更为广泛波长的光能, 并且在高温或强光时合成较多的类胡萝卜素以保 护细胞免受伤害 ${ }^{[11]}$. 有报道称铜绿微囊藻在较高温度时 光合速率和可溶性蛋白含量升高 ${ }^{[12]}$, 从而在能量利用和 物质积累上获得竞争优势. 在第九天时三个温度下两种 藻细胞密度比值均出现拐点, 可见此时两种藻从自由生 长阶段进人竞争利用有限资源的阶段 ${ }^{[7]}$, 拐点后两者密 度比值出现转折或者差距进一步拉大, 微囊藻优势体现 得更为明显. 本试验是在静止培养条件下进行的, 如有不 适当的扰动以及新鲜培养液的补充就有可能削弱微囊藻 的优势 ${ }^{[6,13]}$, 有报道显示在 $25^{\circ} \mathrm{C}$ 恒化培养时, 培养液日稀 释倍数大于 0.65 的条件下四棘栅藻 (Scenedesmus quadri$\operatorname{cauda}$ ) 在与微囊藻的竞争中占优势 ${ }^{[13]} .18^{\circ} \mathrm{C}$ 时微囊藻与 小球藻细胞密度比值出现先减后增的现象, 可能在于该 温度下微囊藻延滞期较长, 但在指数期其生长速度快于 小球藻, 从而经过一段时间后微囊藻仍然有可能占优势, 这与报道的太湖中微囊藻水华在 $18.2^{\circ} \mathrm{C}-32.5^{\circ} \mathrm{C}$ 出现相 吻合 ${ }^{[1]}$. 在实际情况中, 微囊藻生物量达到一定量后水表 可见水华的出现还取决于当时的水文、气象等多种条

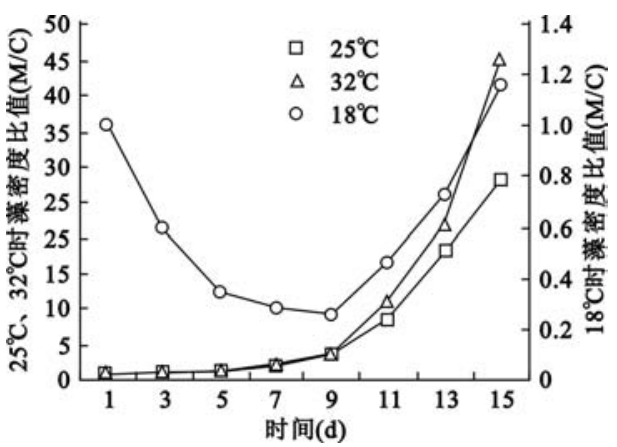

图 3 不同温度下混合培养微囊藻与小球藻 的细胞密度比值(微囊藻/小球藻)

Fig. 3 Mixed culture algae cell density ratio between Microcystis Aeruginosa and Chlorella pyrenoidosa under different temperature $(\mathrm{M} / \mathrm{C})$ 件 ${ }^{[14]}$, 微囊藻水华的出现指示着微囊藻在湖泊藻类生态系统中已经取得绝对优势.

\section{2 藻细胞内 Chla 的荧光强度}

以往的检测结果显示藻类活体细胞 Chla 苂光强度与其含量成较好的线形关系 ${ }^{[15-17]}$. 本实验的统计结 果显示 $32^{\circ} \mathrm{C}$ 时 (图 4a) 纯培养对照与混合培养组中微囊藻 $\mathrm{Chla}$ 苂光强度差异不显著 $(P>0.05$ ), 但该温度 时的小球藻 Chla 菼光强度是纯培养对照略高于混合培养组 $\left(P<0.05\right.$ ). 在 $25^{\circ} \mathrm{C}$ 和 $18^{\circ} \mathrm{C}$ 时 (图 $4 \mathrm{~b}, \mathrm{c}$ ), 纯培 养微囊藻或小球藻 Chla 苂光强度均高于混合培养组中该藻 Chla 的苂光强度 $(P<0.05)$. 可见竞争对 Chla 苂光有一定影响, 种间竞争的影响大于种内竞争. $32^{\circ} \mathrm{C}$ 时混合培养体系中就 $\mathrm{Chla}$ 苂光而言, 小球藻对微囊 藻影响不明显, 而微囊藻对小球藻有一定影响. 在本试验温度范围内 $\left(18-32^{\circ} \mathrm{C}\right)$ 提高温度加快了色素合成 速率, 增大了光能获得效率和能量转换速率 ${ }^{[18]}$, 绿藻细胞中 Chla 苂光强度随着温度的升高有所增加, 而蓝 藻细胞中 Chla 苂光强度随着温度升高却有所下降. 这种现象的解释需要结合细胞密度比值的检测结果综 合考虑. 微囊藻在较高温度时, 其细胞增殖加速, 数量明显增加, 但是 Chla 合成速率的增加无法跟上细胞分 裂速率的增加, 使得单个细胞中的 Chla 含量减少. Chla 荧光强度的减弱还可能与蓝藻中色素蛋白复合体的 结构有关, 因为蓝藻能随着环境的变化来改变藻胆蛋白的组成和含量, 以更有效地利用光能 ${ }^{[19]}$. 较高的温 度也有利于藻蓝素的合成, 其含量增加可能会在空间位置上对 Chla 苂光产生一定的屏蔽作用 ${ }^{[20]}$, 影响检 测结果, 但是由于藻蓝素可以吸收并传输光能给 Chla 进行光合作用, 因而在利用光能方面具有一定的补偿 

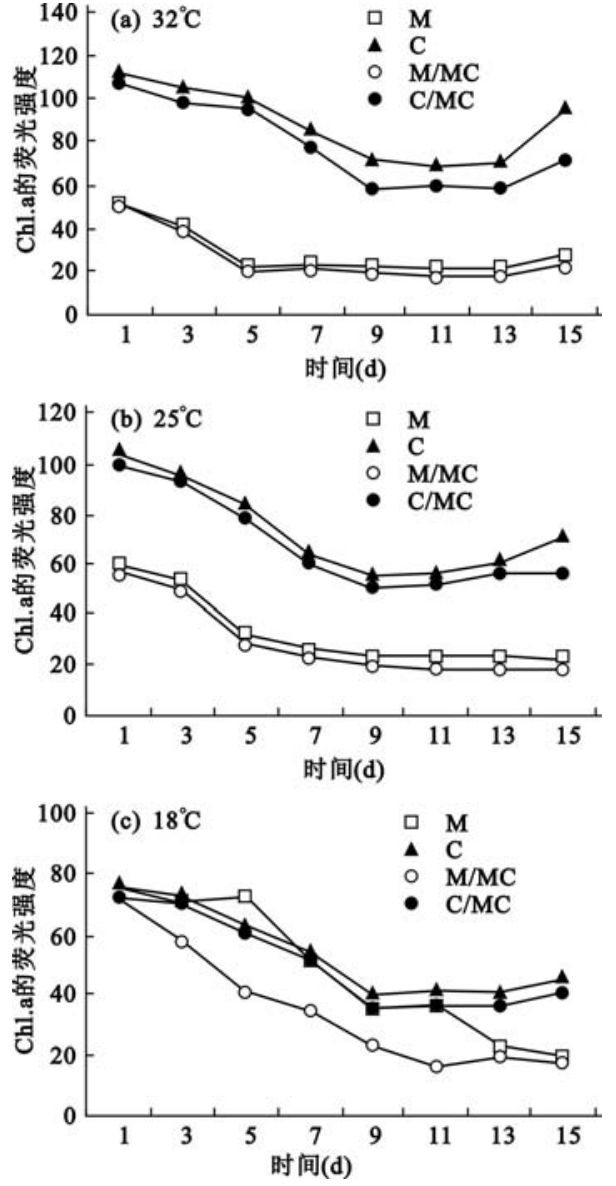

图 4 藻细胞 $\mathrm{Chla}$ 的菼光强度

$$
\left(\mathrm{a}: 32^{\circ} \mathrm{C} ; \mathrm{b}: 25^{\circ} \mathrm{C} ; \mathrm{c}: 18^{\circ} \mathrm{C}\right. \text { ) }
$$

$(\mathrm{M}$ : 纯培养微囊藻; $\mathrm{C}$ : 纯培养小球藻, $\mathrm{M} / \mathrm{M}$; $\mathrm{C}$ : 混合培养微囊藻; $\mathrm{C} / \mathrm{MC}$ : 混合培养小球藻)

Fig. 6 Chla fluorescence intensity of single algae cell under $32^{\circ} \mathrm{C}$ ( a ) $; 25^{\circ} \mathrm{C}$ ( b ) and $18^{\circ} \mathrm{C} \mathrm{(c)}$

( M:Pure culture Microcystis aeruginosa;

C:Pure culture Chlorella pyrenoidosa;

M/MC: Microcystis aeruginosa in mixed culture;

C/MC: Chlorella pyrenoidosa in mixed culture)

效应. 这可能是微囊藻在竞争中的一种策略,即首先在 细胞数量上占据足够的生态空间, 形成优势. 从图中苂 光强度变化趋势可见, 同一温度下随着培养时间的后移 细胞组分有所变化,加之种内或种间竞争对细胞活性的 影响, 细胞内色素含量随之变化 ${ }^{[18]}$, 从而 Chla 苂光强度 也随之变化 (图 4-6). 对于混合培养体系中两种藻细 胞各自的色素苂光强度与其竞争能力间的关系, 有必要 结合蓝藻中的藻蓝素苂光以及绿藻中的 Chlb 苂光做进 一步研究.

\subsection{FCM 在藻类竞争试验中运用的效果分析}

利用 FCM 技术, 可以对培养液中的每个藻细胞的 生理状态进行逐个检测与分析. 本试验的检测值为 $10^{4}$ 个细胞的平均值, 每秒的细胞流量在 $10^{3}$ 个左右, 如此大 量的样本分析数据用传统的实验方法很难快速获取, 因 而 FCM 的检测结果具有大样本的统计学意义, 使得 FCM 检测结果更加可信. 本试验选用 SSC 差异和 FL3 (Chla 苂光) 信号强弱准确地区分了外形大小极其相似 的铜绿微囊藻和蛋白核小球藻, 其效果优于 FSC ( Forward-angle scatter, 前向散射角反映细胞大小差别) 和 FL3 组合. FCM 配有精密的光学系统和液流系统, 加上 灵敏的检测器和系统分析软件,这些特点都为研究混合 培养体系中不同藻类之间的竞争规律提供了良好的实 验平台. 本试验中 FCM 同时检测出不同藻类细胞在混 合培养体系中各自的 Chla 菼光强度,这是传统方法所无 法进行的, 其快速原位的检测方式反映了活体细胞的真 实状况. 对于同一类藻的不同品系, FCM 也能根据其细 微差别从混合体系中准确地分选, 若配用菼光底物或苂 光标记物, 流式细胞仪还能检测多项生理指标 ${ }^{[21-23]}$, 目 前已有用 FCM 研究藻菌共生关系以及病毒与藻寄生关 系的报道, 可见 FCM 在淡水浮游生物相互作用的研究 中将被更为广泛地运用 ${ }^{[24-26]}$.

\section{3 结论}

(1) 在不同温度条件下 $\left(18^{\circ} \mathrm{C}, 25^{\circ} \mathrm{C}\right.$ 和 $\left.32^{\circ} \mathrm{C}\right)$, 随着 温度的升高混合培养体系中微囊藻优势逐渐明显.

(2) 在不同温度条件下 $\left(18^{\circ} \mathrm{C}, 25^{\circ} \mathrm{C}\right.$ 和 $\left.32^{\circ} \mathrm{C}\right)$, 纯培 养对照组以及混合培养体系中蛋白核小球藻细胞内 Chla 苂光强度随着温度的升高有所增强, 而铜绿微囊藻细胞内 Chla 苂光强度随着温度的升高却有所减弱, 这可能是微囊藻适应高温, 迅速增加细胞数量, 并逐步形成优势的竞争策略之一.

\section{4 参考文献}

[1] Chen Y W, Qin B Q, Katrin Teubner, et al. Long-term dynamics of phytoplankton assemblages: Microcystis- domination in Lake Taihu, a large shallow lake in China. Journal of Plankton Research, 2003 ,25 (4) : $445-453$. 
[2] Dokulil M, Chen W, Cai Q. Anthropogenic impacts to large lakes in China: the Tai Hu example. Aquatic Ecosystem Health and Management, $2000,3: 81$ - 94.

[3] 孔繁翔,高光. 大型浅水富营养化湖泊中蓝藻水华形成机理的思考. 生态学报, 2005,25(3): 589 -595 .

[4] Holm N P, Armstrong. Role of nutrient limitation and competition in controlling the population Asterionella forosa and Microcstis aeruginosa semicontinuous culture. Limnology and Oceanography, 1981, 26: 672 -684 .

[5] Akira Kuwata, Tatsuo Miyazaki. Effects of ammonium supply rates on compeition between Microcystis novacekii (Cyanobacteria) and Scenedesmus quadricauda (Chlorophyta) : simulation study. Ecological Modelling, $2000,81-87$.

[6] Kaoru Takeya, Akira Kuwata, Makoto Yoshida, et al. Effect of dilution rate on competitive interactions between the cyanobacterium Microcystis novacekii and the green alga Scenedesmus quadricauda in mixed chemostat culture. Journal of Plankton Research, 2004,26(1):29 - 35 .

[7] 陈德辉, 章宗涉, 刘永定等. 微囊藻和栅藻共培养实验及其竞争参数的计算. 生态学报, 1999,19(6): $908-913$.

[8] Corzo A F, Jimenez Gomez F, Gordillo R, et al. Synechococcus and Prochlorocccus-like populations detected by flow cytometry in a eutrophic reservoir in summer. J Plankton Research, 1999,21:1575 - 1581.

[9] Huisman J, Jonker R R, Zonneveld C, et al. Competition for light between phytoplankton species: experimental tests of mechanistic theory. Ecology, 1999,80(1):211 - 221 .

[10] 华汝成. 单细胞藻类的培养与利用. 北京:农业出版社, $1980: 101$.

[11] Paerl H W, Tucker J, Bland P T. Carotenoid enhancement and its role in maintaining blue-green(Microcystis aeruginosa) surface blooms. Limnol Oceanogr, 1983,28:847 - 857.

[12] 王习达, 吴国荣, 陆长梅等. 两种温度条件下苯酚对铜绿微囊藻大型变种生长的影响. 广西植物, $2004,24(3): 281-285$.

[13] 张民, 民小丽, 蒋丽娟等. 两种外源性磷及振荡对铜绿微囊藻生长的影响. 应用与环境生物学报, $2002,8: 507-510$.

[14] Oliver R L, Ganf G G. Freshwater blooms. In: Whitton BA \& Potts M ed. The Ecology of Cynobacteria. The Netherlands: Kluwer Academic Publishier, 2000: 149 - 194.

[15] Graziano L M, Geider R J, Li W K W, et al. Nitrogen limitation of North Atlantic phytoplankton: Analysis of physiological condition in nutrient enrichment experiments. Aquat Microb Ecol,1996,11:53 - 64 .

[16] Li W K W, Zohary T, Yacobi Y Z, et al. Ultraphytoplankton in the eastern Mediterranean Sea: towards deriving phytoplankton biomass from flow cytometric measurements of abundance, fluorescence and light scatter. Mar Ecol Progr Ser,1993,102:79 - 87.

[17] Veldhuis M J W \& Kraay G W. Application of flow cytometry in marine phytoplankton research: Current applications and future perspectives. Sc Mar, 2000,64(2):121-134.

[18] 陈慈美, 周慈由, 郑爱榕等. 中肋骨条藻增殖的环境制约作用 $\mathrm{Fe}$ ( III ) 与 $\mathrm{N} 、 \mathrm{Mn}$ 、光、温交互作用对藻生 化组成的效应. 海洋通报, $1996,15(2): 37-42$.

[19] 韩博平,韩志国,付 翔. 藻类光合作用机理与模型. 北京:科学出版社, 2003:17-23.

[20] Gantt E. Pigment protein complexes and the concept of the photosynthetic unit: chlorophyll complexes and phycobilisomes. Photosynt Res, 1996,48:47 - 53.

[21] Crosbie N D, Teubner K, Weisse T. Flow-cytometric mapping privides novel insights into the seasonal and vertical distributions of freshwater autotrophic picoplankton. Aquatic Microbiol Ecology, 2003 ,33 (1) :53 66.

[22] Natasha M Franklin, Jennifer L, Richard P Lim. Development of multispecies algal bioassays using flow cy- 
tometry. Environmental Toxicology and Chemistry, 2004,23(6) : $1452-1462$.

[23] Jackie L Collier. Flow cytometry and the single cell in Phycology. J Phycology, 2000,36: 628 - 644.

[24] Corina PD Brussaard. Optimization of procedures for counting viruses by flow cytometry. Applied and Environmental Microbiology, 2004,70(3):1506 - 1513.

[25] Stefan Andreatta, Manfred M Wallinger, Thomas Posch, et al. Detection of subgroups from flow cytometry measurements of heterotrophic bacterioplankton by image analysis. Cytometry, 2001,44(3):218 - 225.

[26] Nicholas D Crosbie, Matthias Pöckl, Thomas Weisse. Rapid establishment of clonal isolates of freshwater autotrophic picoplankton by single-cell and single-colony sorting. Journal of Microbiological Methods, 2003, $55: 361-370$. 